\title{
Revitalization of the Force Simulator and Adequacy of Joystick Loads Compatible with Toucan T-27 and Super Toucan AT-29 Aircraft
}

\section{Thiago Augusto Rochetti Bezerra ${ }^{1^{*}}$, Antônio Carlos Shimano² and Paulo Roberto Pereira Santiago ${ }^{3}$}

${ }^{1}$ Graduate Program in Operational Human Performance/PPGDHO, Brazilian Air Force, University of the Air Force, Brazil

${ }^{2}$ Department of Biomechanics, University of São Paulo, Brazil

${ }^{3}$ School of Physical Education and Sport of Ribeirão Preto, University of São Paulo, Brazil

\begin{abstract}
The innovative design of a "Force Simulator for the EMB 312 T-27- Toucan" aircraft was developed through the regular research support from FAPESP, $N^{\circ} 2005 / 51519-0$. The result of this research project can be observed through the acquired patent (Privilege of Innovation/ Registration number: 0000220708555664), 7 (seven) published articles (3 with impact factor), and presentations at National and International congresses as a result of research using this equipment. In the period from 2008 to 2017, this Simulator was widely used in the post-doctoral research of the beneficiary of the project, entitled "Analysis of Intracranial Pressure (ICP) using a non-invasive method in Brazilian Air Force Aviators submitted to increases in Gz in flight and simulator force tests", FAPESP n²014/21803-7. Research partnerships were carried out with researchers from the Medical School of Ribeirão Preto/USP, Institute of Physics of São Carlos/ USP, Technological Institute of Aeronautics, and Center of Aerospace Research in Microgravity of the Pontifical Catholic University of RS, providing great study possibilities in the area. Aiming at the continuity of the experiments, we are requesting through FAPESP Regular Aid support for "Revitalization of the Force Simulator and suitability of compatible loads on the T-27 Toucan and AT-29 Super toucan aircraft". The objective of this current project is to carry out more advanced research in a sector still little explored in Brazil (aerospace), but with increasing worldwide expansion, providing continuity to studies revitalizing the Force Simulator. For this we count on our experience of more than thirteen years of research in the field, highlighting the results already acquired up to the present moment, and will receive scientific support from researchers and highly specialized institutions. Thus, we won an Regular assistance and financial support for this project to the São Paulo Research Foundation (FAPESP).
\end{abstract}

\section{Keywords}

Force simulator, Load cell, Aerospace, Gz load, Accelerometers

\footnotetext{
*Corresponding author: Thiago Augusto Rochetti Bezerra, Post-Graduation Program in Operational Human Performance/PPGDHO, Brazilian Air Force, University of the Air Force, Brazil Accepted: March 14, 2019; Published: March 16, 2019

Copyright: (C) 2019 Bezerra TAR, et al. This is an open-access article distributed under the terms of the Creative Commons Attribution License, which permits unrestricted use, distribution, and reproduction in any medium, provided the original author and source are credited.

Bezerra et al. Int J Astronaut Aeronautical Eng 2019, 4:027
}

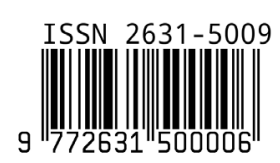




\section{Introduction}

\section{History of the force simulator}

The Air Force Simulator (SF) of the T-27 Aircraft was built between 2005 and 2008, in the Precision Workshop of the Medical Faculty of Ribeirão Preto, University of São Paulo. The objective of this equipment is to develop a simulator of forces exerted in the joystick of the aircraft EMB 312 Toucan T-27, for evaluation of forces and physical training of pilots and cadets. The equipment created simulates, through a joystick and spring system, mechanical forces very close to the actual forces applied to the joystick during the execution of a maneuvers/acrobatics.

A system composed of four load cells connected to a signal logger enabled quantification of the forces as a function of time in the simulator. This signal encoder module was responsible for filtering and digitizing signals from the load cells. Software integrated with the signal logger was able to store and provide information about the force and moment registers. The signal conditioning was able to provide the varying voltage gain steps, such that the signals from the transducers could be faithfully represented after data collection. The signals applied to each load cell were digitized for subsequent processing in the application. The equipment captured the signals of variation of this applied voltage on the load cells connected to the springs and provided a voltage value corresponding to this action. The simulator can be used as training to strengthen muscle groups required in acrobatic flights and as a tool for physical evaluation and scientific research in Pilots and Aviator Cadets of the Brazilian Air Force during the years 2008 to 2017.

The construction of this equipment was part of the Master Dissertation (defended in 2008) of the Beneficiary of the current project, under the guidance of Prof. Dr. Antônio Carlos Shimano from FMRP/USP, Bioengineering Laboratory.

The Force Simulator was constructed by means of the FAPESP Regular Research project, PROCESS No.2005/51519-0, denominated "DESIGN AND DEVELOPMENT OF AN EMB 312- TOUCANT- 27 AIRCRAFT FORCE SIMULATOR".

Prior to the dissertation defense, the equipment underwent calibration tests and was screened by the most experienced pilots in the Brazilian
Air Force, belonging to the Aerial Demonstration Squadron "SMOKE SQUADRON".

\section{Use of the force simulator for research}

The force of gravity is altered many times during an acrobatic flight. A pilot's body weight may increase by 5 to 6 times for a few seconds. Consequently the mechanical forces performed by the pilot on the lever, pedals, and joystick are increased. Failure to prepare physically for the flight can lead to muscle and joint injuries and decrease flight safety, increasing the risk of collision with other aircraft, and falls. The force simulator aims to reduce the risk of injury and increase flight safety, by improving the muscle strength of pilots in the movement of the joystick. The principal characteristics of the force simulator are the training of specific muscle groups recruited in flights and the simulation of loads applied to the joystick during the execution of simulated maneuvers.

This equipment is extremely useful in research at the Air Force Academy. Many of the physiological alterations that pilots undergo during flight can be analyzed in the force simulator without any risk. Studies have already been performed to monitor heart rate, blood pressure, and intracranial pressure using, primarily, physical exertion in the FS.

\section{Innovations Suggested in the Revitalization of the Force Simulator}

The innovations of this equipment can be characterized with regard to the joint adaptability of the Force Simulator to two aircraft, these being the T-27 and AT-29.

At the time of its construction, the Aerial Demonstration Squadron (ADS) used the T-27 aircraft in its presentations (Figure 1). However, from 2013 the ADS began to use the new version of this aircraft (Figure 2) Although the AT-29 aircraft has internal dimensions identical to those of the T-27, regarding ejector seat size, size of the joystick, and dimensions of the joystick, there is a great difference in the loads exerted on the joystick. The AT-29 aircraft has an empty weight of $3200 \mathrm{~kg}$, compared to $1810 \mathrm{~kg}$ for the T-27 aircraft. The maximum speed also differs (AT-29 = $590 \mathrm{~km} / \mathrm{h}, \mathrm{T}-27=448 \mathrm{~kg}$ ). The AT-29 aircraft has load factors: $+7 \mathrm{G} /-3.5 \mathrm{G}$ while the T-27 has load factors: $+6 \mathrm{G} /-2.5 \mathrm{G}$ All of these differences mean the AT-29 aircraft has an extremely heavy joystick 


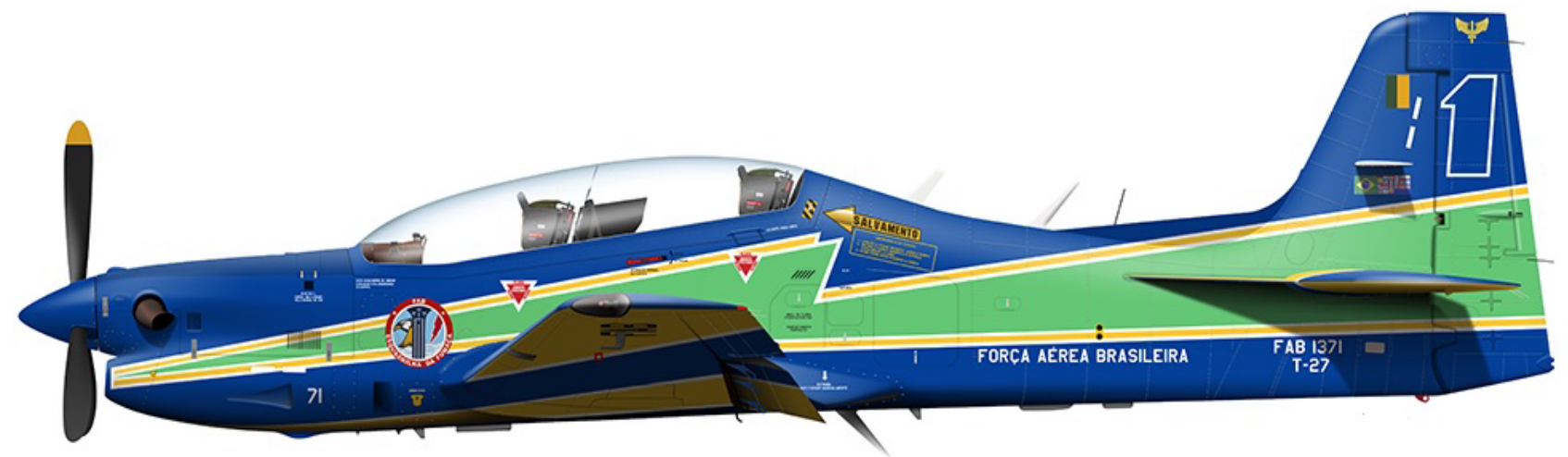

Figure 1: TOUCAN T-27 Aircraft. Source: BAF.

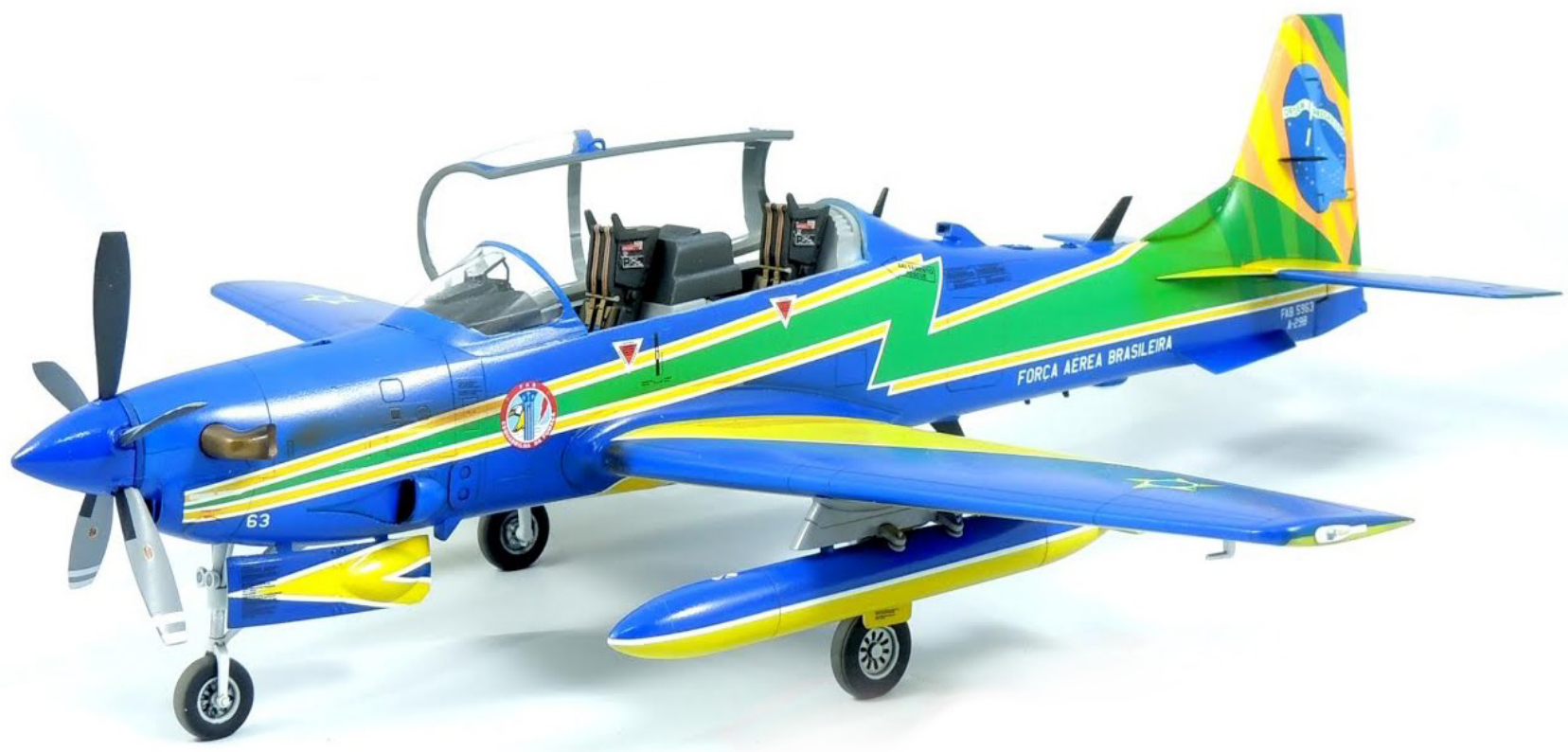

Figure 2: SUPERTOUCAN AT-29 Aircraft. Source: BAF.

of $47 \mathrm{kgf}$ (maximum weight required), against 30 $\mathrm{kgf}$ (maximum weight required in the T-27 aircraft) (AERONAUTICAL COMMAND, 2017).

The Brazilian Air Force (BAF) also uses AT-29 aircraft in the fight against drug trafficking and for border watch. With this in mind, this project aims not only to refine the simulation analysis of the loads required by the T-27 aircraft, but also to simulate the forces in the AT-29 aircraft. Thus, this simulator may be useful in the future for the BAF, with real chances of being produced in larger quantities.

\section{Equipment to be Purchased in the Revital- ization of the Simulator}

\section{New load cells}

The "S" beam load cells are designed with mechanical overload protection of $500 \% \mathrm{FE}$ in tension and compression. This unique design offers an additional level of protection and functionality, which is typically an additional cost option for the majority of load cells. In case of overload, the load cell is protected by an exclusive locking design. When the overload is removed, the load cell is unlocked and returns to normal operation. The stainless steel sensor element makes this load cell one of the most durable models available on the market.

\section{Capacity from 11 to $340 \mathrm{~kg}$}

Characteristics: Excitation: $10 \mathrm{Vdc}, 15 \mathrm{~V}$ maximum, Output: $3 \mathrm{mV} / \mathrm{V} \pm 0.25 \%$ of full scale, Calibration: point scanning of 5 points, In tension at $0 \%, 50 \%, 100 \%, 50 \%, 0 \%$, Linearity: $\pm 0.03 \%$ of full 
scale, Hysteresis: $\pm 0.02 \%$ of full scale, Repeatability: $\pm 0.01 \%$ of full scale, Zero Balance: $\pm 1 \%$ of full scale, Creep in 20 minutes: $\pm 0.03 \%$ of full scale, Operating Temperature: -18 to $65^{\circ} \mathrm{C}\left(0\right.$ to $\left.150{ }^{\circ} \mathrm{F}\right)$, Compensated temperature: -18 to $65^{\circ} \mathrm{C}$ ( 0 to 150 $\left.{ }^{\circ} \mathrm{F}\right)$, Thermal effects: Zero: $\pm 0.0027 \% \mathrm{FS} /{ }^{\circ} \mathrm{C}$ Span: $\pm 0.0014 \% \mathrm{rdg} /{ }^{\circ} \mathrm{C}$, Safe overload: $500 \%$ capacity compression and tension, Final overload: $500 \%$ of capacity, Bridge resistance: 350 (c) nominal, Level of protection: IP67, Material: 17-4 element PH SS, Aluminum side cover, Electrical connection: $6 \mathrm{~m}$ (20 ') 4-conductor 24 AWG shielded cable.

Justification for the purchase: Although the Force simulator already uses load cells, these need to be replaced (they were positioned in 2007, and are already demonstrating flaws), and with modern technology. The quoted models meet the need for modernization of the simulator.

\section{Floor replacement}

Five meters (5) of aluminum, to replace the NAVAL wood floor.

Justification for the purchase: Five meters (5) of aluminum, to replace the (current) NAVAL wood floor. Lighter and longer lasting material.

\section{Panel gauges/Controllers}

The series gauges together with a 4-digit, 9-segment display offer unequaled accuracy in signal conditioning. Additional features include microprocessor-based operation, 5 A SPDT (optional) dual relays and analog output isolated or not at intervals of 0 to $10 \mathrm{VCC}, 0$ to $20 \mathrm{~mA}$, or 4 to $20 \mathrm{~mA}$ ranges, which can be used to retransmit the value on the display or as a proportional control output. The DP25 series includes models with process outputs (DC, voltage and current), strain gages, thermocouples and RTDs.

Justification for the purchase: This equipment will update the values provided by the load cells and accelerometers, modernizing the simulator.

\section{Accelerometer}

The accelerometer is extremely durable. It is constructed of high strength aluminum, featuring reinforced strain relief, robust screw mounting and epoxy seal, and uses reliable components.

Justification for the purchase: This equipment was not used in the first version of the Force Simulator. However, its use could greatly increase the reliability of studies, providing precise information in the processes of evaluation of accelerations in the joystick.

\section{New data acquisition module}

A high-speed USB 2.0 data acquisition module with thermocouple/voltage input (fully compatible with USB 1.1 and USB 2.0 ports). This standalone module is powered directly by the USB port, while an external power supply (optional) can be used. All configurable options (including individual channel input type and range) are programmable using the software. The OM-DAQ-USB-2401 has programmable voltage inputs, ranging from $30 \mathrm{mV}$ to $10 \mathrm{~V}$, full scale. Compact and modular packaging ensures ease of use in a variety of applications. Units can be DIN rail or wall mounted with the included hardware, or easily operated on a bench. All analog inputs can be measured sequentially at approximately 1 ms per channel.

Justification for the purchase: A total of 1000 samples per second can be taken, divided into all active channels. This is fundamental equipment in data acquisition, especially in accelerations in the joystick. This equipment was not used in the first version of the force simulator.

\section{Extractor PIN Type C $3.0 \times 100 / 2.0 \times 50$}

Justification for the purchase: Extractor Pin Type C $3.0 \times 100 / 2.0 \times 50$, will be used internally next to the system of compression springs.

\section{Calibrated compression springs kit, ISO 10243 Standard}

Justification for the purchase: A calibrated compression spring kit can simulate the mechanical forces of the T-27 and AT-29 aircraft, during the Gz load simulation applied to the joystick.

\section{STUDY 1}

Electromyographic analysis in upper limbs of the $1^{\text {st }}$ Air Force Instruction Squadron of the Brazilian Air Force submitted to a stress test in an Force Simulator compatible with Toucan T-27 and Super toucan AT-29 aircraft.

The position adopted by pilots is complex. At the same time they are required to maintain the body for the effects of increasing Gravitational load $(\mathrm{Gz})$ and use the senses of sight, touch and executing hand on the joystick, and feet on the pedals according to the necessary actions, all this 
respecting the joint boundaries in a manner to avoid pain. These movements place high demand on skeletal muscles and the entire circulatory system [1,2].

The health status of a pilot is not independent of professional activity. Health hazards associated with acrobatic flight may have negative aspects, since the pilots do not receive specific preparation for certain situations [3].

Physical training should be directed at injury prevention and pilot-specific training. Few situations expose the human organism to a great variation of pressures and muscular efforts as the military flight of maneuvers and acrobatics [2-4].

In military flight, it is common for the aircraft to exceed the gravity acceleration constant $(1 \mathrm{Gz}+)$.

At the Brazilian Air Force Academy (AFA), pilots are subjected to $\mathrm{Gz}+$ load variations five times greater than gravity.

The present study used electromyographic methods to determine the specific training needs of the Brazilian Air Force Academy (AFA) pilots.

Electromyography is a method of recording the electrical potential changes of a muscle, allowing access to the patterns of muscular electrical activity, and providing research on likely synergies, and the predominance of muscles in determined patterns of movement $[5,6]$.

\section{Material and Methods}

Data collection was performed at the Laboratory of Biomechanics and Motor Control (LABIOCOM) EEFERP-USP. Surface electromyography was used as it provides greater comfort for the volunteer and is easy to manage for the researcher $[7,8]$. The sampling frequency for the surface EMG signal was in the order of $2000 \mathrm{hz}$. The electrodes used were Delsys-trigno wireless, following the recommendations of the SENIAM (Surface Electromyography for the Non-Invasive Assessment of Muscles). The distance between the electrodes was $20 \mathrm{~mm}$ from center to center. Prior to placement of the electrodes the following procedures were performed: Cleaning the skin where the electrode was to be placed; removal of hairs; and light scraping of the skin for removal of dead cells. The electromyograph used was of the brand Delsys-trigno wireless. The following muscles were analyzed: Flexor carpi radialis, extensor carpi ulnaris, brachial biceps, brachial triceps, anterior deltoid, posterior deltoid, upper trapezius, pectoralis major, infra-spinal, and latissimus dorsi (Figure 3). Figure 4, Figure 5, Figure 6 and Figure 7 show the execution of the analyzed movements.

Twelve male flight instructors of the Air Force Academy participated voluntarily in this study,

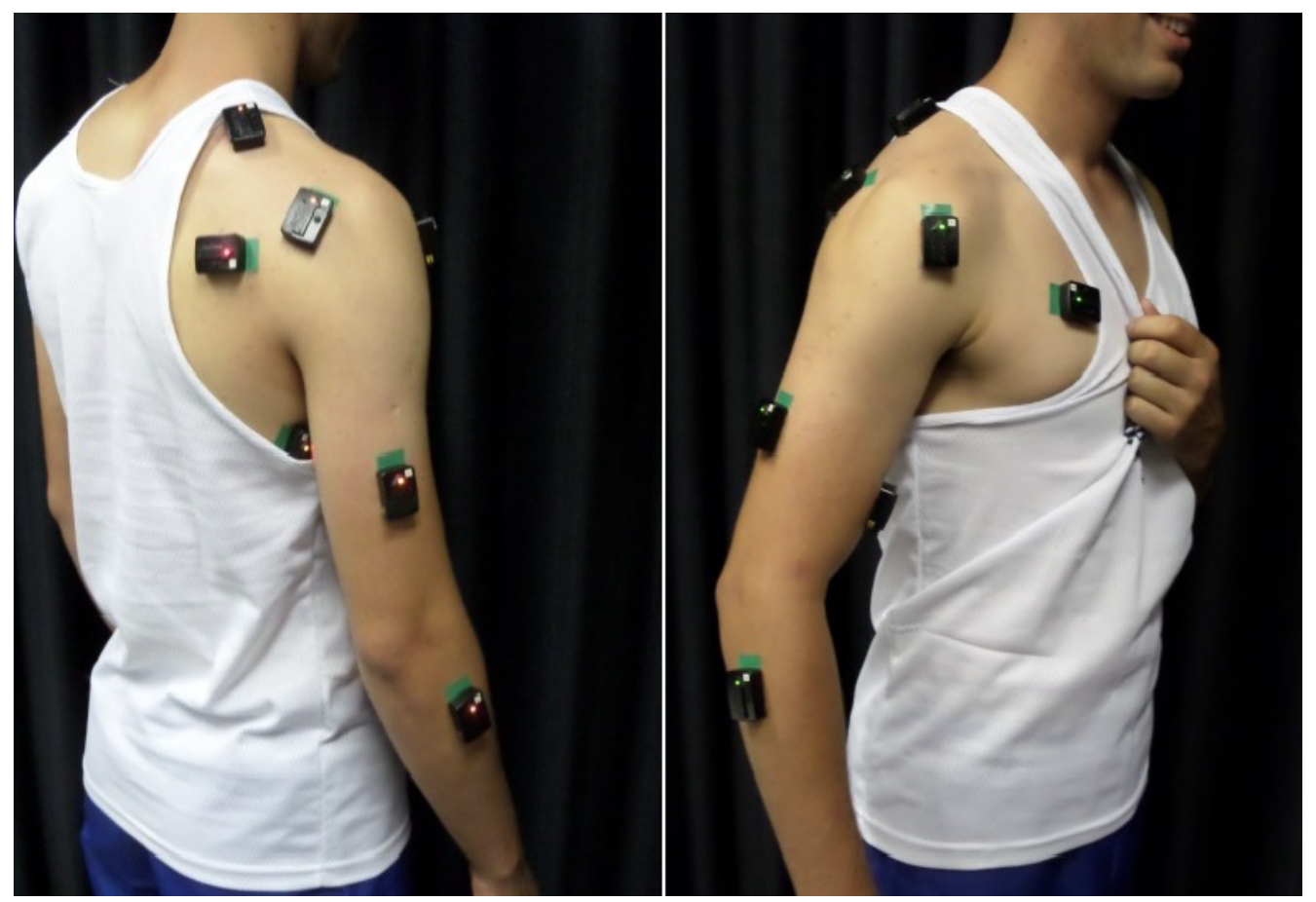

Figure 3: Placement of the electrodes. 


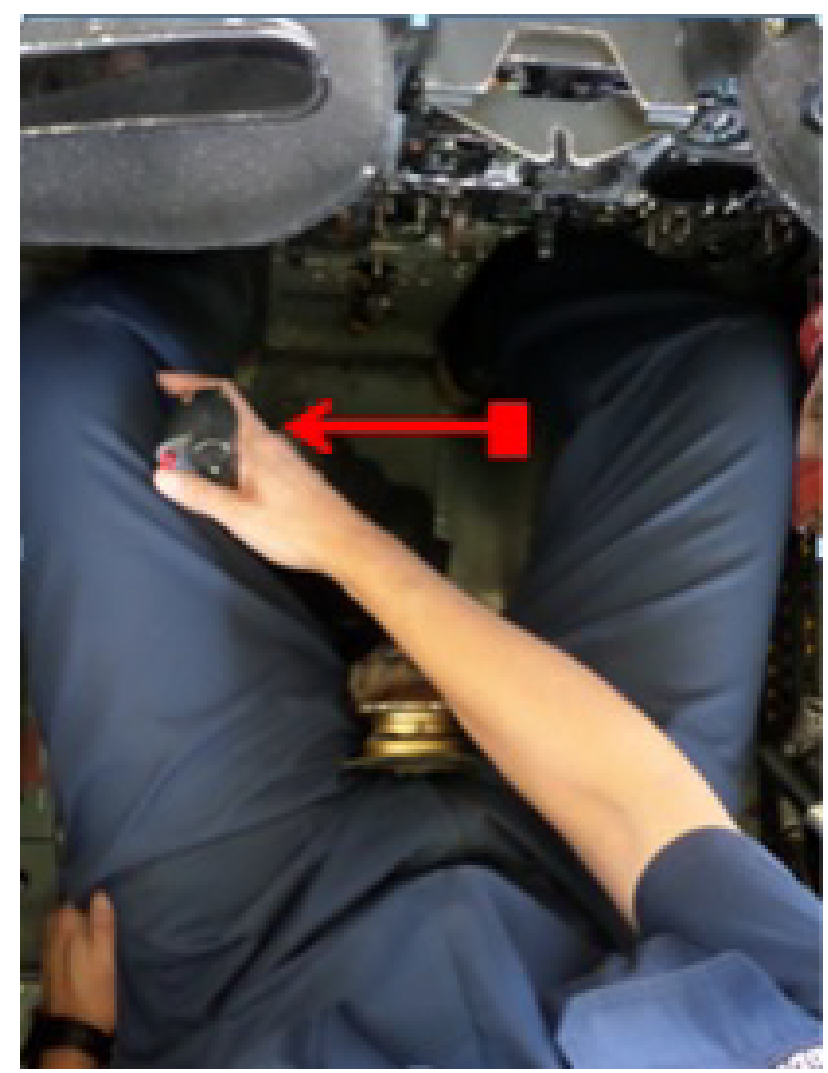

Figure 4: Rot. Left.

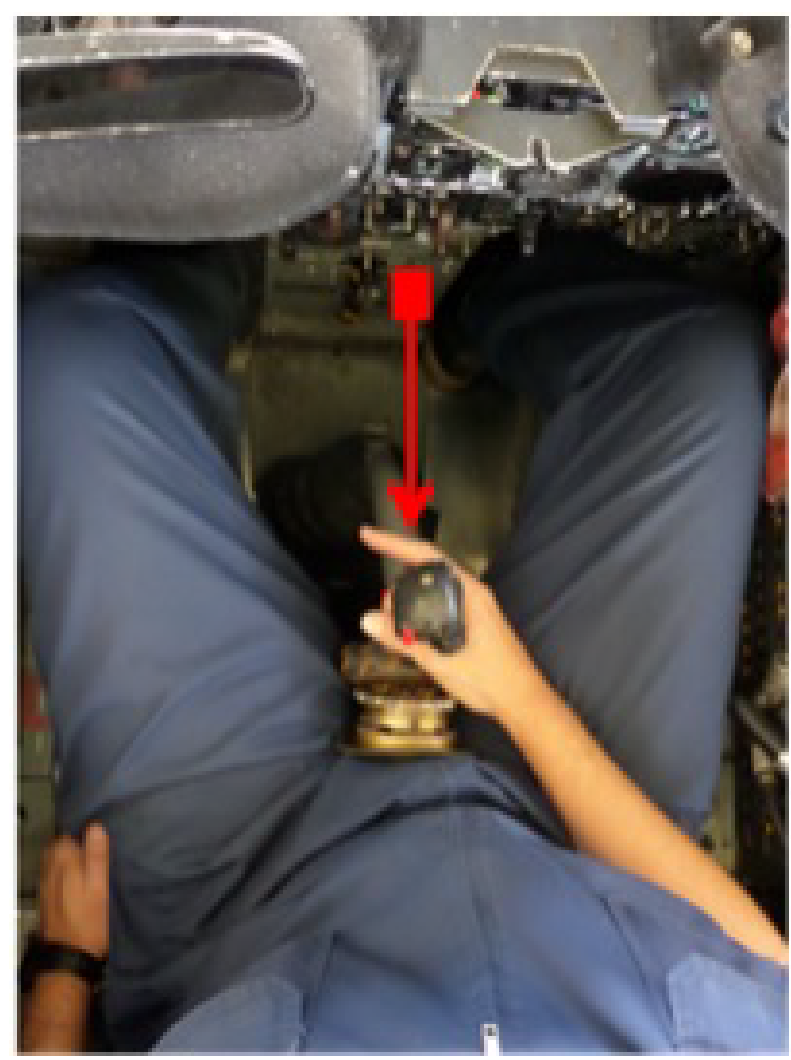

Figure 5: The Back.

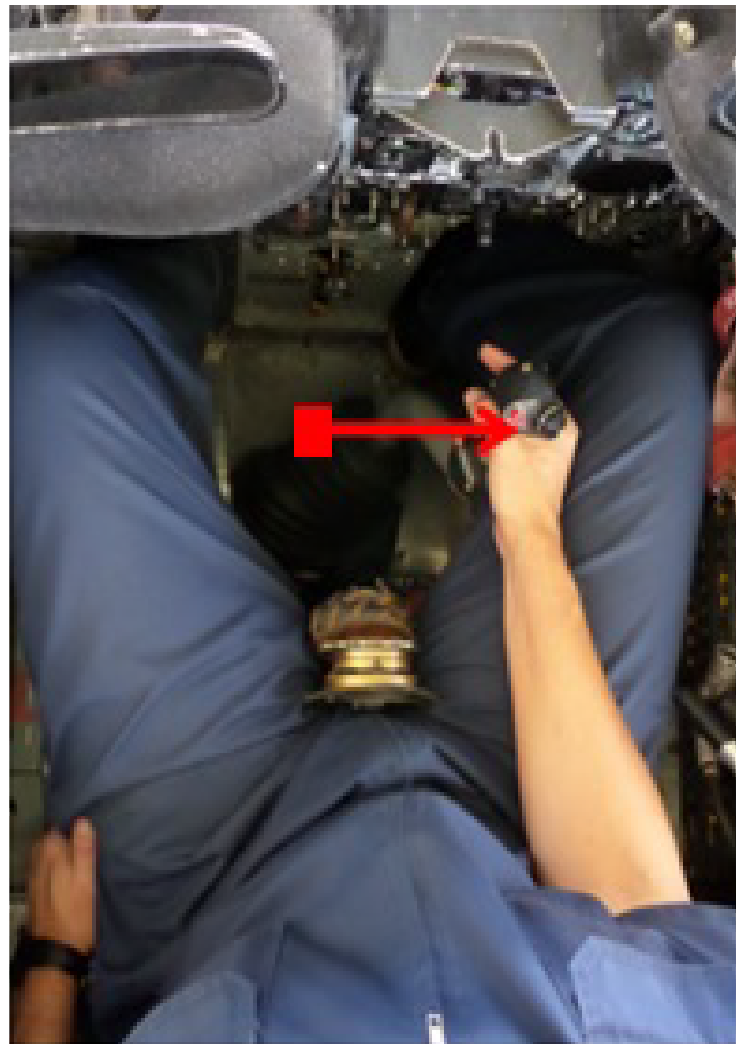

Figure 6: Rot. right.

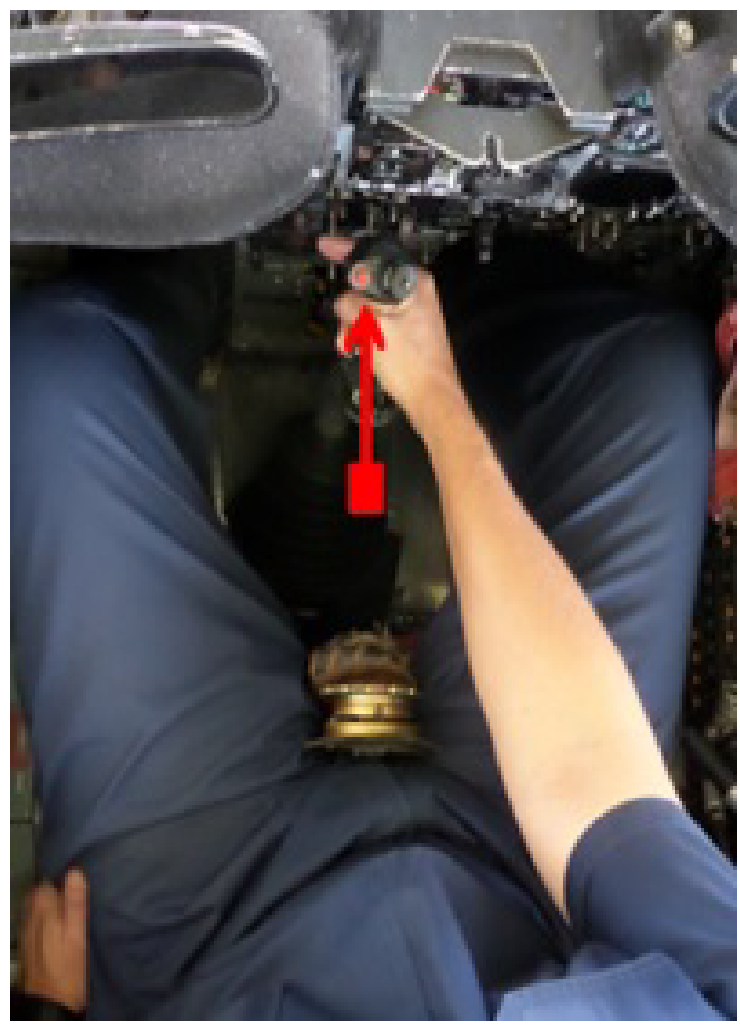

Figure 7: The Front. 


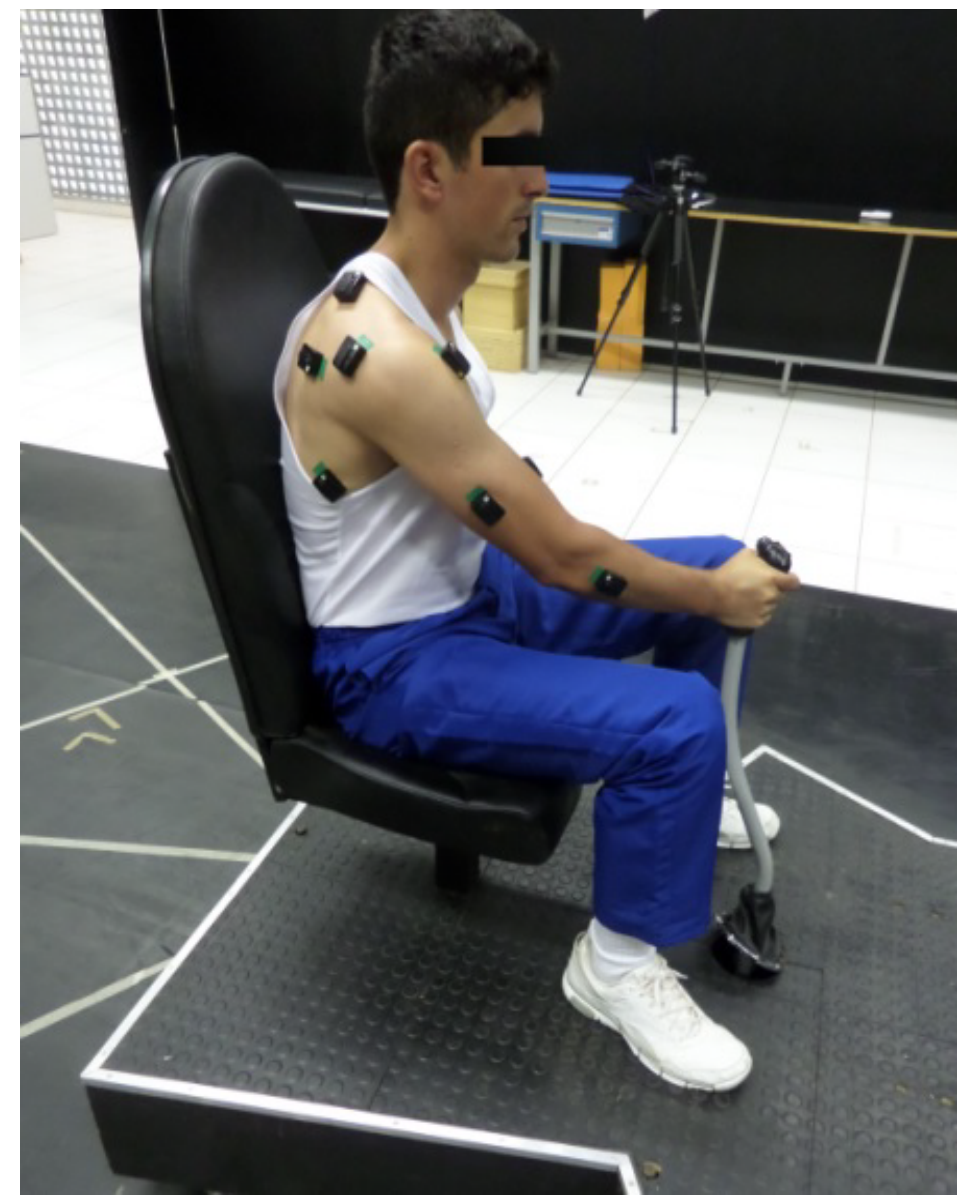

Figure 8: Lateral view of the Force Simulator.

without any cardiorespiratory or musculoskeletal impairment or other conditions that could impede or prejudice the subject during performance of the experimental procedures. The descriptive data (mean \pm standard deviation) showed a mean age of $28.8 \pm 2.5$ years, weight $68.9 \pm 7.4 \mathrm{Kg}$, and height $1.73 \pm 0.04 \mathrm{~cm}$.

The equipment and instruments used in this project were:

(1) Force Simulator Prototype for the Airplane EMB 312 T-27 "Tucano" and AT 29 SUPERTOUCAN.

(2) Electric Signal Acquisitor of the Prototype for the Force Simulator Prototype.

(3) Delsys- trigno wireless electromyograph and its signal capture system.

Figure 8 shows a lateral view of the force simulator prototype.

The prototype joystick was connected at its lower end by 4 joints, one for each prime direction of the simulator. For calibration of the forces exerted on the joystick steel springs were used, connected at their ends to a load cell. The load cells perform measurement of the forces.

The storage and processing of the forces data were transmitted by each load cell as a function of time, performed by the electrical signal Acquisitor coupled to a computer. The electrical signal Acquisitor recorded the signals acquired by the load cells in graphics of force applied as a function of time.

The measures of the maximum time of isometric contraction were performed in the movements: (1) The front; (2) The back; (3) Rolling right; and (4) Rolling left, respectively. A maximum time of 60 seconds was established for each movement, based on the duration of a real stunt. The time interval between movements was set at 30 seconds, which is equivalent to the time spent in actual training performed in the aircraft in flight.

The isometric evaluations were performed in the Force Simulator calibrated to a force of 30 kgf. This force is equivalent to the maximum force applied during the execution of stunts in the aircraft subjected to a gravitational force equal to $5 \mathrm{Gz}$. 
Table 1: Analysis of the Mean Electromyographic Activation (RMS - mV).

\begin{tabular}{|l|l|l|l|l|}
\hline & The Front & The Back & Rot. int. & Rot. Ext. \\
\hline Flexor Carpi Radialis & 7.440 & 123.349 & 81.1849 & 182.141 \\
\hline Extensor Carpi Ulnaris & 37.092 & 25.752 & 41.724 & 39.354 \\
\hline Brachial Biceps & 9.659 & 54.043 & 440.263 & 20.926 \\
\hline Brachial Triceps & 52.088 & 18.723 & 40.771 & 62.312 \\
\hline Anterior Deltoid & 162.280 & 9.045 & 72.617 & 121.446 \\
\hline Posterior Deltoid & 105.173 & 110.030 & 13.536 & 545.028 \\
\hline Upper Trapezius & 8.709 & 59.506 & 9.955 & 125.662 \\
\hline Pectoralis Major & 6.005 & 7.517 & 225.504 & 9.693 \\
\hline Infra-Spinal & 78.904 & 53.972 & 16.035 & 175.009 \\
\hline Latissimus Dorsi & 18.550 & 54.997 & 48.475 & 44.353 \\
\hline Total & 485.904 & 516.938 & 990.068 & 1325.928 \\
\hline MEAN & 48.590 & 51.693 & 99.006 & 132.592 \\
\hline SD & 52.393 & 39.652 & 84.534 & 157.593 \\
\hline CV & 1.078 & 0.7670 & 1.069 & 1.188 \\
\hline
\end{tabular}

The RMS-Root Mean Square of the signal was analyzed through the electromyographic amplitude of each of the selected muscles in the front, the back, External Rotation, and Internal Rotation movements.

The RMS values were smoothed in the program Delsys-trigno wireless, exported to the format ".Txt" and passed to the MatLab program, where a specific routine was used to remove the mean and peak values for each trial in each condition.

The RMS data (Root Mean Square) found through the routine created in the MatLab program were normalized by the peak of the EMG signal of each volunteer.

To analyze the data distribution, the ShapiroWilk normality test was applied. For statistical analysis of the electromyographic peak data the ANOVA test was used. For the data of the mean electromyography amplitude we used the ANOVA multifactorial test. The Action add-in for the program Excel (Microsoft USA) was utilized.

\section{Results}

There was a higher mean electromyographic activation of the Anterior Deltoid muscle in the front movement (RMS-mV = 162.280); the Flexor Carpi Radialis muscle in the back movement (RMS$m V=123.349)$; the Brachial Biceps Muscle in the Internal Rotation movement (RMS-mV $=440.263$ ); and the Posterior Deltoid Muscle in the External
Rotation Movement (RMS-mV = 545.028). Through the analysis of the mean electromyographic activation, there was greater muscle activation in the External Rotation movement (RMS-mV = 1325.928) followed by the Internal Rotation (RMS$m V=990.0680)$, the back (RMS-mV = 516.93), and the front movements (RMS-mV $=485.904$ ).

The mean values of electromyographic activation are presented in Table 1.

The analysis of peak electromyographic activation showed that the highest values were presented for the Anterior Deltoid muscle (RMS-mV = 334.234) in the front movement; the Flexor Carpi Radialis muscle in the back movement (RMS-mV = 407.969), the Brachial Biceps muscle in the Internal Rotation movement (RMS-mV = 314.587), and the Posterior Deltoid Muscle in the External Rotation movement (RMS-mV = 1277.229). The analysis of the peak electromyographic activation showed greater muscle activation in the External Rotation movement (RMS-mV = 3598.876), followed by the Internal Rotation (RMS-mV = 1598.964), the back (RMS-mV = 1371.476), and the front movements (RMS-mV = 993.856).

The values of the Peak electromyographic activation are shown in Table 2.

\section{Discussion}

There was higher mean electromyographic activation for the Anterior Deltoid muscle in the 
Table 2: Analysis of the Peak Electromyographic amplitude (RMS - microVolts).

\begin{tabular}{|l|l|l|l|l|}
\hline & The Front & The Back & Rot. int. & Rot. Ext. \\
\hline Flexor Carpi Radialis & 21.693 & 407.969 & 268.368 & 572.583 \\
\hline Extensor Carpi Ulnaris & 55.785 & 55.431 & 126.201 & 95.449 \\
\hline Brachial Biceps & 16.758 & 262.987 & 314.587 & 94.332 \\
\hline Brachial Triceps & 83.028 & 37.861 & 73.188 & 173.620 \\
\hline Anterior deltoid & 334.234 & 27.925 & 238.549 & 432.402 \\
\hline Posterior deltoid & 203.173 & 145.888 & 47.771 & 1277.229 \\
\hline Upper trapezius & 21.403 & 133.301 & 19.160 & 369.121 \\
\hline Pectoralis major & 13.459 & 17.230 & 264.760 & 19.093 \\
\hline Infra-spinal & 206.431 & 156.022 & 39.222 & 470.104 \\
\hline Latissimus dorsi & 37.886 & 126.859 & 207.154 & 94.938 \\
\hline Total & 993.856 & 1371.476 & 1598.964 & 3598.876 \\
\hline MEAN & 99.385 & 137.147 & 159.896 & 359.887 \\
\hline SD & 110.384 & 121.370 & 110.908 & 375.015 \\
\hline CV & 1.110 & 0.884 & 0.693 & 1.042 \\
\hline
\end{tabular}

front movement (RMS-mV = 162.280); the Flexor Carpi Radialis muscle in the back movement; (RMS$\mathrm{mV}=123.349)$ the Brachial Biceps Muscle in the Internal Rotation movement (RMS-mV $=440.263$ ), and the Posterior Deltoid Muscle in the External Rotation movement (RMS-mV = 545.028). Through the analysis of the mean electromyographic activation, there was greater muscle activation in the External Rotation movement (RMS-mV = 1325.928) followed by the Internal Rotation (RMS$m V=990.0680)$, the back (RMS-mV = 516.93), and the front movements (RMS-mV $=485.904$ ).

The analysis of the peak electromyographic activation demonstrated that the highest values were presented for the Anterior Deltoid muscle (RMS-mV = 334.234) in the front movement; the Flexor Carpi Radialis muscle in the back movement (RMS-mV = 407.969), the Brachial Biceps Muscle in the Internal Rotation movement (RMS-mV = 314.587), and the posterior Deltoid Muscle in the External Rotation movement (RMS-mV = 1277.229). The analysis of the peak electromyographic activation demonstrated greater muscle activation in the External Rotation movement (RMS-mV = 3598.876), followed by the Internal Rotation (RMS$\mathrm{mV}=1598.964)$, back (RMS-mV = 1371.476), and front movements (RMS-mV $=993.856$ ).

The overload is increased when the pilot is subjected to $\mathrm{Gz}+$ force during acrobatic maneuvers [9]. This force is external and compresses the pilot in the seat, increasing the effort necessary during movement of the limbs. This overload can cause pain and injury [2-4]. The acceleration force is proportional to the velocity and inversely proportional to the angle of the curve that the aircraft performs. As it is a compressive force that can reach a value of $5 \mathrm{Gz}+$ during acrobatic maneuvers, it causes an increase in body weight and body segments by up to 5 times (according to the value of $\mathrm{Gz+}$ ), exposing pilots to severe joint and muscular overloads that may cause injuries [10].

Adaptations are highly necessary, and the lack of specific physical training can reduce unequal flight conditions, causing postural alterations in pilots $[11,12]$.

In this way, the pilots can have favorable conditions to pilot, without greater physical overloads and with satisfactory conditions to eject in case of extreme necessity $[1,11,13]$.

Knowledge of the morphological characteristics of BAF pilots is of great value in the development of new aircraft designs and in adaptations of aircraft already manufactured or acquired.

\section{Acknowledgement}

The Authors would like to acknowledge to the financial support for "Project of Revitalization of the Force Simulator and adequacy of joystick loads compatible with TOUCAN T-27 and SUPERTOUCAN 
AT-29 aircraft" number 2018/02106-4, to the São Paulo Research Foundation (FAPESP)

\section{References}

1. Mierau A, Girgenrath $M$, Bock $O$ (2008) Isometric force production during changed-Gz episodes of parabolic flight. Eur J Appl Physiol 102: 313-318.

2. Albery WB (2004) Acceleration in other axes affects $+G z$ tolerance: Dynamic centrifuge simulation of agile flight. Aviat Space Environ Med 75: 1-6.

3. Whinnery T, Forster EM (2013) The $+G z$-induced loss of consciousness curve. Extrem Physiol Med 2: 19.

4. Tripp LD, Warm JS, Matthews G, Chiu P, Werchan P, et al. (2006) $+G z$ acceleration loss of consciousness: Time course of performance deficits with repeated experience. Hum Factors 48: 109-120.

5. Paz GA, Maia M de F, Lima VP, Oliveira CG, Bezerra E, Simão R, et al. (2012) Maximal exercise performance and eletromiography responses after antagonist neuromuscular proprioceptive facilitation: A pilot study. J Exerc Physiol 8: 11-25.

6. Ramírez A, Garzón DA (2008) Sensitivity analysis for the positioning of electrodes in surface electromiography (semg). Rev Fac Ing la Univ Antioquia 46: 10.

7. Bellomo R, Zoccolella S, Lapenna F, Lecce B, Cologna $D$, et al. (2013) Electromiography as useful test in the differential diagnosis of neuromuscular diseases only after clinical examination. Clinical Neurophysiology 124: e205-e205.

8. Merletti Roberto, Parker P (2004) Electromyography: Physiology, Engineering and Nonivasive Applications. Published by John Wiley \& Sons, Inc., Hoboken, New Jersey, 1: 1-506.

9. Stevenson AT, Scott JPR (2014) +Gz Tolerance, with and without muscle tensing, following loss of anti-g trouser pressure. Aviat Sp Environ Med 85: 426-432.

10. Melek WW, Lu Z, Kapps A, Cheung B (2002) Modeling of dynamic cardiovascular responses during G-transition-induced orthostatic stress in pitch and roll rotations. IEEE Trans Biomed Eng 49: 1481-1490.

11. Newsom BD, Goldenrath WL, Winter WR, Sandler H (1977) Tolerance of females to $+G(z)$ centrifugation before and after bedrest. Aviation space and Environmental Medicine 48: 327-331.

12.Schlegel TT, Wood SJ, Brown TE, Harm DL, Rupert $\mathrm{AH}$ (2003) Effect of 30-min $+3 \mathrm{Gz}$ centrifugation on vestibular and autonomic cardiovascular function. Aviat Space Environ Med 74: 717-724.

13. Han W-Q, Hu W-D, Dong M-Q, Fu Z-J, Wen Z-H, et al. (2009) Cerebral hemodynamics and brain functional activity during lower body negative pressure. Aviat Space Environ Med 80: 698-702. 